\title{
Synthesis and Photoluminescence Studies of ZnO Films Using Vapor Phase Transport Combined With Rapid Heating
}

\author{
Iksoo Ji and Jae-Young Leem* \\ Department of Nanoscience and Engineering, Inje University, Gimhae 50834, Republic of Korea
}

\begin{abstract}
The vapor phase transport (VPT) process is usually used for the growth of one-dimensional nanostructures rather than for film deposition. In this study, for the first time, we report on the fabrication and optical propeties of $\mathrm{ZnO}$ film produced by VPT combined with rapid heating. The X-ray diffraction results showed that the intensity of the $\mathrm{ZnO}(002)$ peak was the largest, which indicated that the c-axis orientation of the $\mathrm{ZnO}$ grains was perpendicular to the substrate. In the photoluminescence (PL) spectra of the $\mathrm{ZnO}$ film at $12 \mathrm{~K}$, PL peaks were observed at 3.374, 3.362, 3.319, and $3.242 \mathrm{eV}$, which were attributed to free excitons (FXs), excitons bound to neutral donors, donor-acceptor pairs (DAPs), and first-order longitudinal optical phonon replicas of DAPs (DAPs-1LO), respectively. The energy interval between DAPs and DAPs-1LO was almost the same as the longitudinal optical (LO) phonon energy of bulk $\mathrm{ZnO}$ of $\sim 76 \mathrm{meV}$. The PL spectra in the temperature range from 12 to $300 \mathrm{~K}$ shifted to lower energy with an increasing temperature. The activation energy of FX was estimated to be $61 \mathrm{meV}$. This value is similar to the exciton binding energy of $60 \mathrm{meV}$.
\end{abstract}

(Received September 4, 2015; Accepted December 11, 2015)

Keywords: thin films, vapor deposition, optical properties, X-ray diffraction, photoluminescence.

\section{INTRODUCTION}

$\mathrm{ZnO}$ is a direct bandgap $(3.37 \mathrm{eV})$ semiconductor that has attracted attention for use in optoelectronic applications such as light-emitting diodes and laser diodes [1,2]. $\mathrm{ZnO}$ has a high exciton binding energy of $60 \mathrm{meV}$, which is substantially higher than the room temperature thermal energy (26 meV) and the exciton binding energy (25 meV) of GaN. As a versatile material with excellent properties, it is also useful in various other applications, such as catalysts, photovoltaics, sensors, surface acoustic wave devices, piezoelectric transducers and actuators, hydrogen storage, and transparent conductive films [3].

Various techniques, such as chemical vapor deposition [4], molecular beam epitaxy [5], pulsed laser deposition [6], thermal evaporation [7], and spray pyrolysis [8], have been used to prepare $\mathrm{ZnO}$ films. Another technique is the vapor phase transport (VPT) method, which is conventionally not a film deposition technique but rather a growth method for one-dimensional nanostructures. If it could be adapted for the deposition of films, this method could provide the possibility

*Corresponding Author: Jae-Young Leem [Tel: +82-55-320-3716, E-mail: jyleem@inje.ac.kr]

Copyright (c) The Korean Institute of Metals and Materials of mass-producing both nanostructures and epitaxial layers (and even bulk crystals) of high quality and purity. With the VPT method, the morphology of the produced samples depends on the growth conditions, including the position of the substrates, source materials, gas flow rates, substrate temperatures, and difference in vapor supersaturation [9]. However, until now, the heating rate of the furnace has not been considered. In general, the furnace is heated at a rate of $25-50{ }^{\circ} \mathrm{C} \min ^{-1}[10,11]$. In this study, the furnace was heated at a rate of $250{ }^{\circ} \mathrm{C} \mathrm{min}^{-1}$ to fabricate $\mathrm{ZnO}$ films.

We used photoluminescence (PL) spectroscopy to study the optical properties of the $\mathrm{ZnO}$ films at temperatures in the range of 12 to $300 \mathrm{~K}$. In general, PL spectroscopy is one of the most widely used experimental methods to study the optical properties of semiconductors [12]. The PL spectrum of $\mathrm{ZnO}$ generally consists of two main bands. The band in the ultraviolet (UV) region corresponds to the near-band edge (NBE) emission attributed to exciton emission originating from the recombination of free excitons through an exciton-exciton collision process; the other band in the visible region is due to structural defects and impurities related to oxygen vacancies or $\mathrm{Zn}$ interstitials. Low-temperature PL spectroscopy is a sensitive and powerful tool for characterizing electrically active impurities [13]. Moreover, 


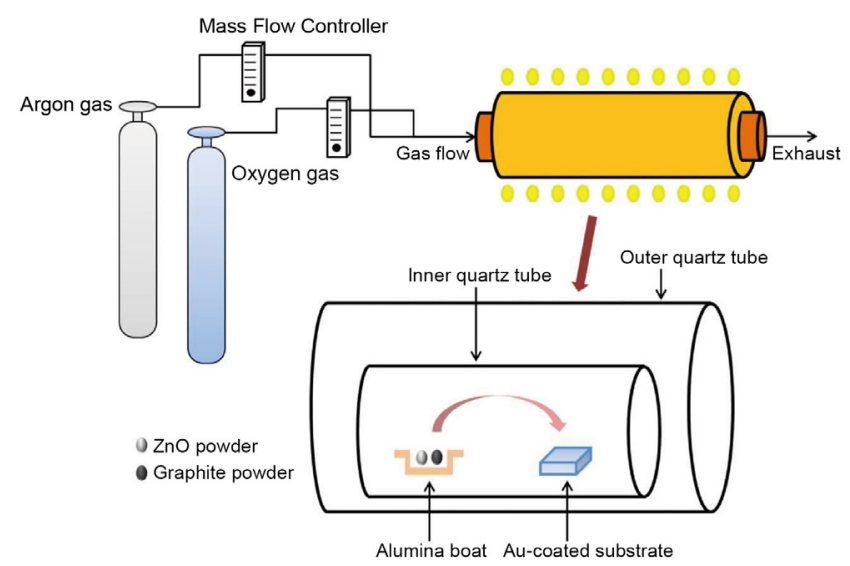

Fig. 1. Experimental setup: schematic of the experimental system.

the low-temperature PL study also reveals the interactions of the nano-confined trapping states and the lattice sub-system. It is important that the synthesized structures have good optical properties for the fabrication of efficient UV-based optoelectronic devices [14]. Hence, we focused on the temperature dependence of the PL spectra with respect to the NBE transition in the range from 12 to $300 \mathrm{~K}$. Spectra pertaining to free excitons (FXs), excitons bound to neutral donors $\left(\mathrm{D}^{0} \mathrm{Xs}\right)$, donor-acceptor pairs (DAPs), and longitudinal optical (LO) phonon replicas of DAPs (DAPs-1LO) were identified and analyzed.

\section{EXPERIMENTAL PROCEDURES}

$\mathrm{ZnO}$ film was produced by the VPT process in a single-zone furnace within a horizontal quartz tube with inner diameter of $38 \mathrm{~mm}$ and length of $485 \mathrm{~mm}$. First, the Si (100) substrate was cleaned using a piranha solution, a mixture of sulfuric acid $\left(\mathrm{H}_{2} \mathrm{SO}_{4}\right)$ and hydrogen peroxide $\left(\mathrm{H}_{2} \mathrm{O}_{2}\right)$, which is commonly used to clean organic residues off substrates. Before growing the $\mathrm{ZnO}$, an $\mathrm{Au}$ (99.9999\%) catalyst layer with a thickness of $5 \mathrm{~nm}$ was deposited onto the Si (100) substrate using ion sputtering. Subsequently, equal amounts of $\mathrm{ZnO}$ powder (99.9995\%, Alfa Aesar) and graphite powder ( 200 mesh, 99.9999\%, Alfa Aesar) were well mixed and placed into a small alumina boat. The boat and Au-coated substrate were placed into a small quartz tube. The small quartz tube was then pushed into a horizontal quartz tube with the source being positioned at the center of the furnace, as shown in Fig. 1. High-purity Ar gas was introduced into the inner quartz tube at a flow rate of $100 \mathrm{sccm}$. Then, the furnace temperature was raised to $900{ }^{\circ} \mathrm{C}$ at a rate of $250{ }^{\circ} \mathrm{C}$ $\min ^{-1}$. When the growth temperature had been reached, oxygen gas was injected into the inner quartz tube at a flow rate of $20 \mathrm{sccm}$. The reaction lasted for about $60 \mathrm{~min}$. After the growth phase, the oxygen-gas supply was cut off, and then the furnace was allowed to cool to room temperature.

The PL properties of the $\mathrm{ZnO}$ film were investigated employing a $\mathrm{He}-\mathrm{Cd}$ laser $(325 \mathrm{~nm})$ with $20 \mathrm{~mW}$ excitation power and a $0.75 \mathrm{~m}$ single-grating monochromator with a photomultiplier tube (HAMAMATSU, R928) using the photocount method in a temperature range from 12 to $300 \mathrm{~K}$. The surface morphology was analyzed using a field-emission scanning electron microscope (FE-SEM, HITACHI, S-4800). The crystal phase of the $\mathrm{ZnO}$ film was investigated using an X-ray diffractometer (XRD, PANalytical X'Pert Pro, the

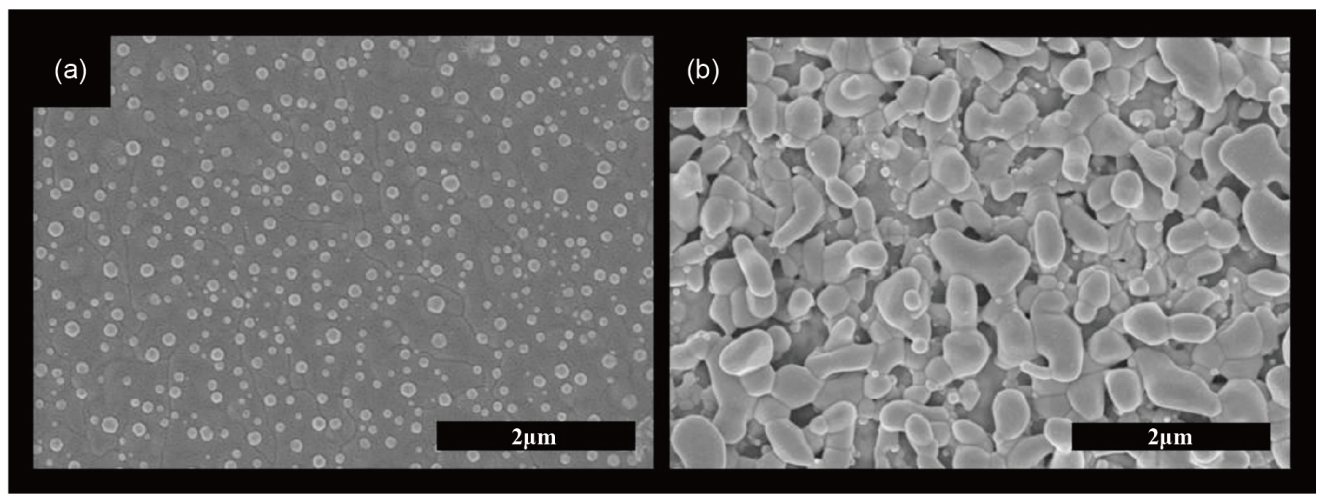

Fig. 2. Microstructures: SEM images of the $\mathrm{ZnO}$ film at (a) the initial stage of the $\mathrm{ZnO}$ growth after 5 min and (b) the final stage of the $\mathrm{ZnO}$ growth. 


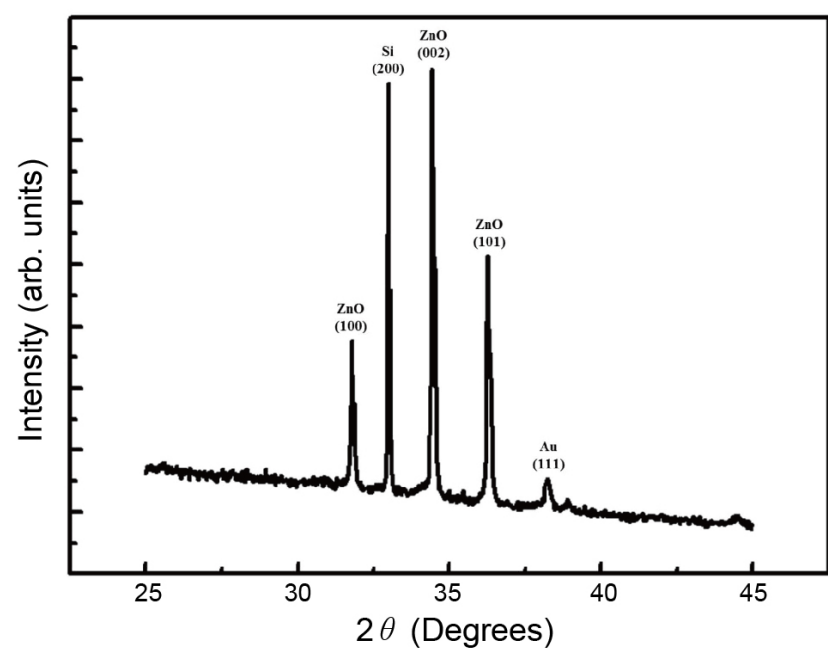

Fig. 3. X-ray diffraction: XRD pattern of the $\mathrm{ZnO}$ film.

Netherlands) with a $\mathrm{Cu}-\mathrm{K} \alpha$ radiation source $(\lambda=0.154056$ $\mathrm{nm}$ ) at an accelerating voltage of $40 \mathrm{kV}$.

\section{RESULTS AND DISCUSSION}

Figure 2(a) indicates dispersed $\mathrm{Au}$ nanoparticles on the $\mathrm{Si}$ substrate at the initial stage of the $\mathrm{ZnO}$ growth after $5 \mathrm{~min}$. The growth of the $\mathrm{ZnO}$ nanostructures is generally controlled by either a vapour-liquid-solid (VLS) mechanism including a metallic catalyst, such as gold, copper, or tin, or a vapour-solid (VS) mechanism without any catalyst [15]. The $\mathrm{Au}$ nanoparticles play an important role in the formation of the $\mathrm{ZnO}$ film in forming nucleation sites. The process involves the reduction of $\mathrm{ZnO}$ powder by carbon to form $\mathrm{Zn}$ and $\mathrm{CO} / \mathrm{CO}_{2}$ vapor in the high-temperature zone. The $\mathrm{Zn}$ vapor is transported and reacts with the Au catalyst on the substrate to form alloy droplets. However, we applied rapid heating for the formation of the $\mathrm{ZnO}$ film. In general, at a rate of $25-50{ }^{\circ} \mathrm{C} \mathrm{min}^{-1}$, the Au nanoparticles agglomerate and form droplets [16]. However, with rapid heating, the $\mathrm{Au}$ nanoparticles did not agglomerate but generated nucleation sites on the substrate. The mixed $\mathrm{ZnO}$ and graphite powders during the carbothermal reaction were in contact at the grain surfaces and then generated $\mathrm{Zn}$ vapor. The processes are described by the following equations [17]:

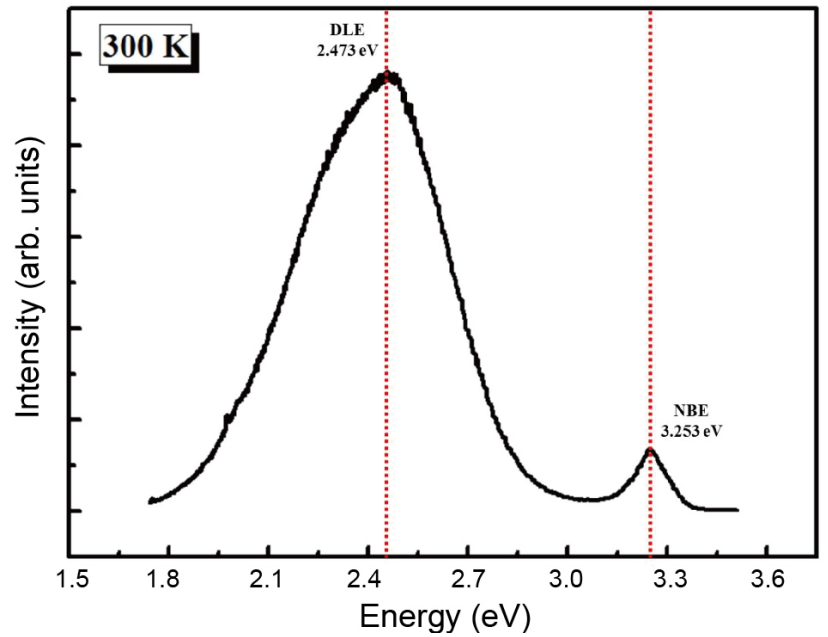

Fig. 4. PL spectroscopy 1: PL spectrum of the $\mathrm{ZnO}$ film over a broad energy range at $300 \mathrm{~K}$.

$$
\begin{aligned}
& \mathrm{ZnO}(\mathrm{s})+\mathrm{C}(\mathrm{s}) \rightarrow \mathrm{Zn}(\mathrm{g})+\mathrm{CO}(\mathrm{g}) \\
& 2 \mathrm{C}+\mathrm{O}_{2} \rightarrow 2 \mathrm{CO} \\
& \mathrm{ZnO}(\mathrm{s})+\mathrm{CO}(\mathrm{g}) \rightarrow \mathrm{Zn}(\mathrm{g})+\mathrm{CO}_{2}(\mathrm{~g}) \\
& \mathrm{ZnO}(\mathrm{s})+(1-x) \mathrm{CO}(\mathrm{g}) \rightarrow \mathrm{ZnO}_{x}(\mathrm{~g})+(1-x) \mathrm{CO}_{2}(\mathrm{~g}) \\
& (0<x<1)
\end{aligned}
$$

The generated $\mathrm{Zn}$ vapor becomes supersaturated at the nucleation sites and forms small clusters. The growth of the $\mathrm{ZnO}$ film is based on the nucleation sites. Therefore, a rapid heating process follows the VS mechanism [18].

Figure 3 shows the XRD pattern of the $\mathrm{ZnO}$ film. Three $\mathrm{ZnO}$ diffraction peaks were observed at $31.8^{\circ}, 34.45^{\circ}$, and $36.28^{\circ}$, which corresponded to the (100), (002), and (101) planes, respectively [19]. The $\mathrm{ZnO}$ film exhibited a strong (002) peak, indicating that the c-axis orientation of the $\mathrm{ZnO}$ grains was perpendicular to the substrate. $\mathrm{ZnO}$ usually grows preferentially oriented along the c-axis under typical growth conditions because the (001) basal plane of $\mathrm{ZnO}$ has the lowest surface energy.

Figure 4 shows the PL spectrum of the $\mathrm{ZnO}$ film at $300 \mathrm{~K}$. PL spectra of $\mathrm{ZnO}$ films are usually composed of an excitonic NBE emission in the UV region and a defect-related deep level (DL) emission in the visible region. The NBE emission 


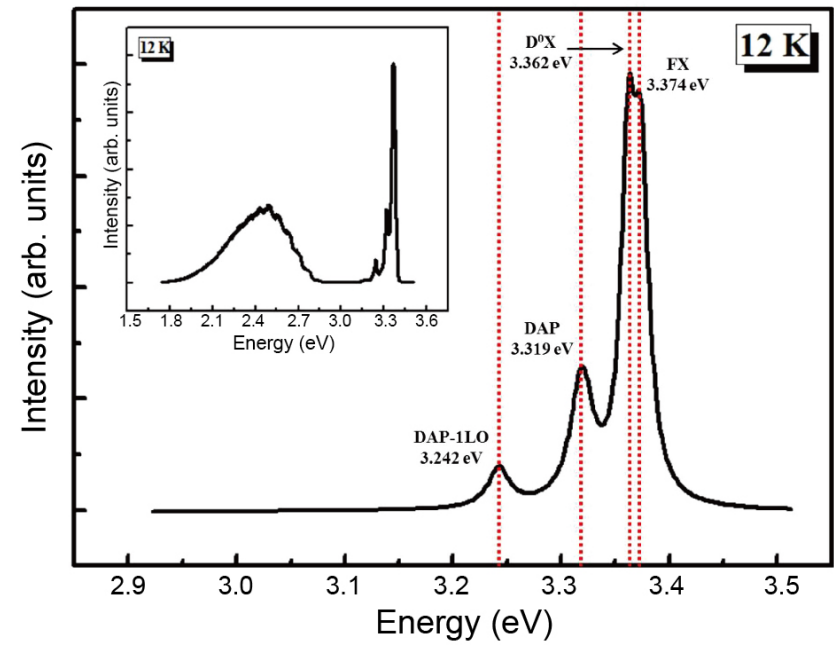

Fig. 5. PL spectroscopy 2: PL spectrum of the $\mathrm{ZnO}$ film in the $\mathrm{NBE}$ region at $12 \mathrm{~K}$. The inset shows this PL spectrum over a broader energy range.

occurs at approximately $3.253 \mathrm{eV}$, which is associated with the recombination of FXs. The strong DL emission occurs at approximately $2.483 \mathrm{eV}$, which is associated with green emission and is related to oxygen vacancies and interstitial zinc atoms in the $\mathrm{ZnO}$ lattice. In general, researchers have reported that singly ionized oxygen vacancies are responsible for the green luminescence of $\mathrm{ZnO}$, which should be related to intrinsic defects of $\mathrm{ZnO}$ films rather than to extrinsic impurities [20].

Figure 5 shows the details of the UV region at $12 \mathrm{~K}$. Four distinct PL peaks appeared in the spectra of the $\mathrm{ZnO}$ film at $3.374,3.362,3.319$, and $3.242 \mathrm{eV}$. The NBE emission peaks at 3.374, 3.362, and $3.319 \mathrm{eV}$ are assigned to FXs, D0Xs, and DAPs, respectively. At the higher energy side of the DOX emission, the emission associated with FXs appears as a shoulder. Due to localization of excitons by impurities, especially due to donors, the FX peak is typically not clearly observed at low temperatures [21]. The peak at $3.242 \mathrm{eV}$ was attributed to DAPs-1LO. The presence of phonon replicas indicates good crystallinity of the $\mathrm{ZnO}$ film [22]. It is estimated that the many strong replicas in the PL spectra result from the low defect concentration compared to other reports [23]. LO phonon replicas of DAPs in $\mathrm{ZnO}$ have usually been identified based on the favorable interval between the DAPs and LO phonon energy $\left(h \omega_{L O}=72 \mathrm{meV}\right)[24]$.

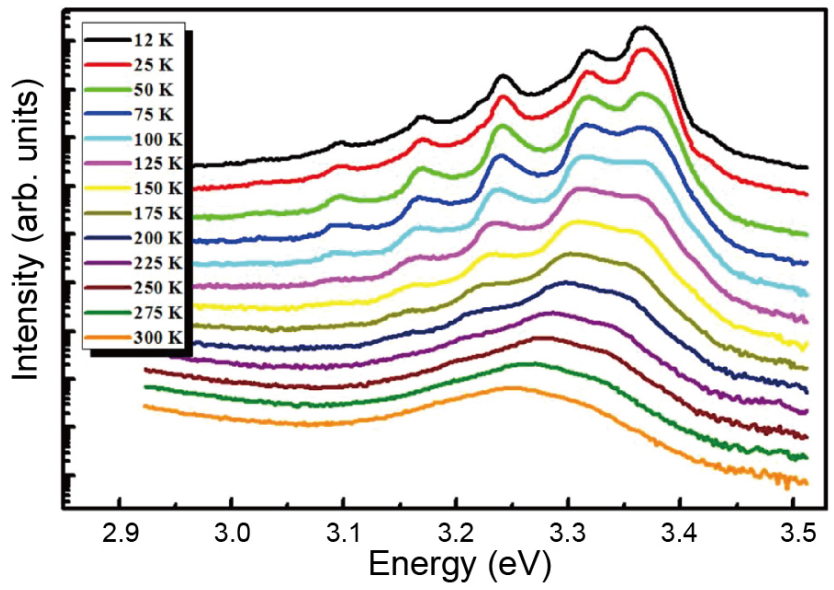

Fig. 6. PL spectroscopy 3: PL spectra of the $\mathrm{ZnO}$ film at different temperatures ranging from 12 to $300 \mathrm{~K}$.

Figure 6 shows the PL spectra at various temperatures in a range from 12 to $300 \mathrm{~K}$. The $\mathrm{PL}$ spectra contained various emission features that changed their intensities and positions with varying temperatures. The intensity of each peak decreased with increasing temperatures because of the thermally induced dissociation of electron-hole pairs. All peaks were redshifted with increasing temperatures. The observed redshift of FXs and $\mathrm{D}^{0} \mathrm{Xs}$ at $30 \mathrm{~K}$ arose because the thermal energy of localized carriers was insufficient to overcome the localization potential at low temperature; hence, they relaxed to lower-lying localized states prior to recombination. At high temperatures with sufficient thermal energy, the redshift arose from the temperature-induced bandgap shrinkage [25]. With an increasing temperature, the DAP emission energy was slightly redshifted. The DAP emission energy is described by [26]

$$
h v=E_{g}-E_{A}-E_{D}+q^{2} / 4 \pi \varepsilon r
$$

$E_{g}$ is the band gap, $E_{A}$ and $E_{D}$ are the acceptor and donor binding energies, respectively, $\varepsilon$ is the dielectric constant, and $r$ is the donor-acceptor distance. With increasing temperature, carriers trapped in DAPs with small distance $r$ are released into the band, and the line is shifted to the low-energy side. The $\mathrm{D}^{0} \mathrm{X}$ emission was dominant at $12 \mathrm{~K}$. However, the FX emission became dominant above $100 \mathrm{~K}$, indicating that it was more probable for the bound excitons to ionize and 


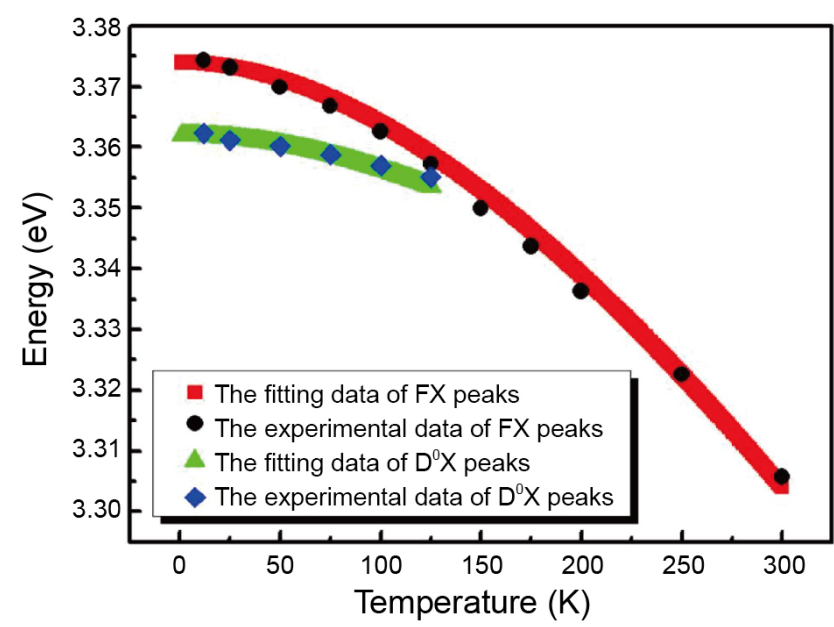

Fig. 7. Analysis of the PL excitations: variations in bandgap energy of the FXs and the D0Xs peaks as a function of temperature. Lines indicate fitting by Varshni's empirical equation.

eventually to transform into FXs as the temperature increased. The UV emission of a $\mathrm{ZnO}$ crystal is usually attributed to interband recombination of electrons and holes in form of excitons. Generally, due to the temperature-induced lattice dilatation and electron-lattice interaction, the interband emission peak energy follows the well-known Varshni formula [27]:

$$
E_{g}(T)=E_{g}(0)-\alpha T^{2} /(\beta+T)
$$

$T$ is the absolute temperature, $E_{g}(0)$ is the band-gap energy at $T=0, \alpha$ and $\beta$ are constants appropriate to $\mathrm{ZnO}$, and $\beta$ is proportional to the Debye temperature $\theta_{D}$. The constants $\alpha$ and $\beta$ were obtained from fitting the PL peaks, as shown in Fig. 7. In the case of the $\mathrm{D}_{0} \mathrm{Xs}$, the constants were determined as $\alpha=0.7 \mathrm{meV}$ and $\beta=600 \mathrm{~K}$, and for FXs, $\alpha=0.5 \mathrm{meV}$ and $\beta=800 \mathrm{~K}$. As is evident from Fig. 7 , the $\mathrm{D}^{0} \mathrm{X}$ peak was dominant in a temperature range of $12-100 \mathrm{~K}$, and the FX peak was dominant above $100 \mathrm{~K}$. The curve determined by the above equation best fit the experimental values, as shown in Fig. 7. The values of $E_{g}(0)$ for the $\mathrm{FX}$ and $\mathrm{D}^{0} \mathrm{X}$ peaks were estimated to be 3.374 and $3.362 \mathrm{eV}$, respectively, which agreed well with the values reported for bulk $\mathrm{ZnO}$; and $\beta$ was also in reasonable agreement with the result of a recent report [28]. It is clear from Fig. 7 that Varshni's equation can

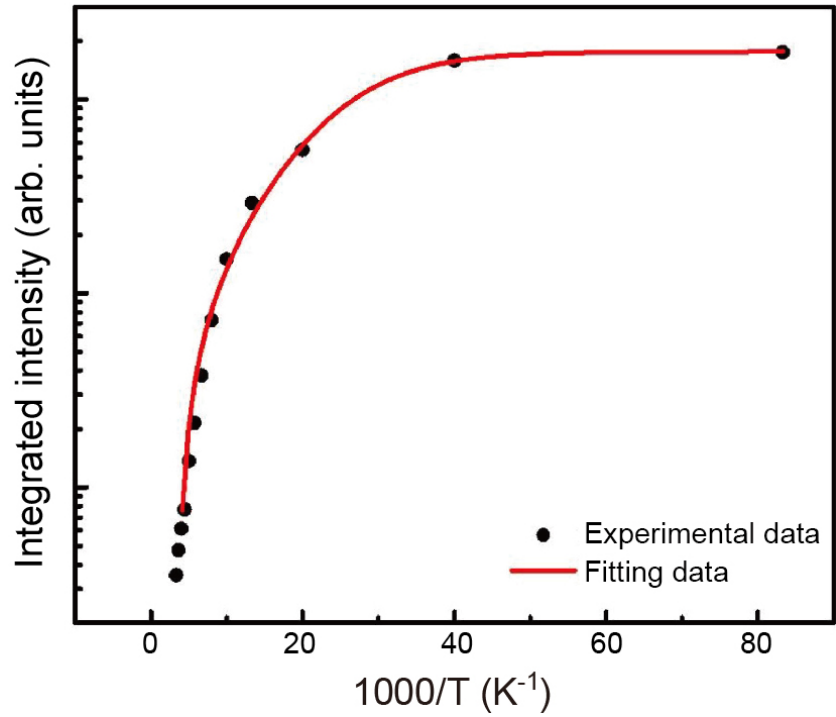

Fig. 8. Arrhenius behavior of FXs: the integrated PL intensity of the FX peaks as a function of the inverse temperature. The line indicates fitting by the Arrhenius equation.

satisfyingly account for the temperature dependent position of the FX peak.

Figure 8 shows the dependence of the integrated intensity of the FX peaks on the reciprocal temperature. The PL intensity decreased appreciably with temperature indicating a negative thermal quenching. The nearly exponential decrease of the PL intensity is attributed to thermal ionization of excitons and thermally activated, non-radiative recombination mechanisms. The overall temperature dependent quenching behavior of the FX intensity is described by the Arrhenius law [29]:

$$
I=I_{0} /\left[1+C \exp \left(-\Delta E_{A} / k_{B} T\right)\right]
$$

$I_{0}$ is the intensity at $0 \mathrm{~K}, C$ is the ratio of the non-radiative transition probabilities, and $\Delta E_{A}$ is the activation energy of the thermal quenching process, $k_{B}$ is the Boltzmann constant and $T$ is the thermodynamic temperature. By fitting the experimental data using Eq. (7), the activation energy is estimated to be $61 \mathrm{meV}$. This value is similar to the exciton binding energy of $60 \mathrm{meV}$ for bulk $\mathrm{ZnO}$ [30]. Therefore, this result suggests that FXs survive at higher temperatures. 


\section{CONCLUSIONS}

In conclusion, the synthesis and optical characterization of $\mathrm{ZnO}$ films using VPT were performed. The XRD results showed $\mathrm{ZnO}$ (100), Si (200), $\mathrm{ZnO}$ (002), $\mathrm{ZnO}$ (101), and $\mathrm{Au}$ (111) diffraction peaks. Among these diffraction peaks, the $\mathrm{ZnO}$ (002) diffraction peak was the most intense. These results correspond to the wurtzite phase, implying the existence of a well-aligned growth direction of the $\mathrm{ZnO}$ film. In the low temperature PL spectrum of the $\mathrm{ZnO}$ film, exciton emissions at 3.374 (FXs), $3.362\left(\mathrm{D}^{0} \mathrm{Xs}\right), 3.319$ (DAPs), and $3.242 \mathrm{eV}$ (DAPs-LO) were observed. The evolution of the different excitonic peaks in the PL spectra was analyzed in a temperature range from 12 to $300 \mathrm{~K}$. With an increasing temperature, the exciton emission was shifted to lower energy (redshift). The observed PL emission energies were fitted numerically using Varshni's empirical equation for the temperature variation of the bandgap. The resulting fitting parameters were $\alpha=0.7 \mathrm{meV}$ and $\beta=600 \mathrm{~K}$ for $\mathrm{D}^{0} \mathrm{Xs}$ and $\alpha$ $=0.5 \mathrm{meV}$ and $\beta=800 \mathrm{~K}$ for FXs. The values of the emission energy at $0 \mathrm{~K}$ for the free- and bound-exciton emissions were estimated to be 3.374 and $3.362 \mathrm{eV}$, respectively. The activation energy of the thermal quenching process was estimated to be $61 \mathrm{meV}$. This value is similar to the exciton binding energy of $60 \mathrm{meV}$ for bulk $\mathrm{ZnO}$.

\section{ACKNOWLEDGEMENT}

This research was supported by Basic Science Research Program through the National Research Foundation of Korea (NRF) funded by the Ministry of Education (No. 2015R1D1A1A01058131).

\section{REREFENCES}

1. M. H. Huang, S. Mao, H. Feick, H. Yan, Y. Wu, H. Kind, E. Weber, R. Russo and P. Yang, Science 292, 1897 (2001).

2. W. I. Park, G. -C. Yi, M. Kim and S. J. Pennycook, $A d v$. Mater. 15, 526 (2003).

3. A. Umar, E. K. Suh and Y. -B. Hahn, J. Phys. D: Appl. Phys. 40, 3478 (2007).

4. P. -C. Chang, Z. Fan, D. Wang, W. -Y. Tseng, W. -A.
Chiou, J. Hong and J. G. Lu, Chem. Mater. 16, 5133 (2004).

5. A. El-Shaer, A. C. Mofor, A. Bakin, M. Kreye and A. Waag, Superlattices Microstruct. 38, 265 (2005).

6. X. M. Fan, J. S. Lian, Z. X. Guo and H. J. Lu, Appl. Surf. Sci. 239, 176 (2005).

7. B. D. Yao, Y. F. Chan and N. Wang, Appl. Phys. Lett. 81, 757 (2002).

8. F. Paraguay D., W. Estrada L., D. R. Acosta N., E. Andrade and M. Miki-Yoshida, Thin Solid Films 350, 192 (1999).

9. A. Bakin, A. C. Mofor, A. El-Shaer and A. Waag, Superlattices Microstruct. 42, 33 (2007).

10. C. Li, G. Fang, J. Li, L. Ai, B. Dong and X. Zhao, J. Phys. Chem. C 112, 990 (2008).

11. Y. Yang, X. W. Sun, B. K. Tay, C. X. Xu, Z. L. Dong and L. Vayssieres, Physica E 40, 859 (2008).

12. Y. Kim, B. Kim and J. -Y. Leem, Korean J. Met. Mater. 53, $751(2015)$

13. F. Khan, S. -H. Baek, N. Ahmad, G. H. Lee, T. H. Seo, E. -K. Suh and J. H. Kim, Met. Mater. Int. 21, 561 (2015).

14. S. Mandal, M. L. N. Goswami, K. Das, A. Dhar, S. K. Ray, Thin Solid Films 516, 8702 (2008).

15. Y. X. Chen, M. Lewis and W. L. Zhou, J. Cryst. Growth 282, 85 (2005).

16. S. R. Hejazi, H. R. M. Hosseini and M. S. Ghamsari, J. Alloys Compd. 455, 353 (2008).

17. S. Kim, G. Nam and J.-Y. Leem, Electron. Mater. Lett. 10, 5, (2014).

18. S. Mandal, K. Sambasivarao, A. Dhar and S. K. Ray, J. Appl. Phys. 106, 024103 (2009).

19. Z. Zhu, T.-L. Chen, Y. Gu, J. Warren and R. M. Osgood, Chem. Mater. 17, 4227 (2005).

20. D. H. Fan, Z. Y. Ning and M. F. Jiang, Appl. Surf. Sci. 245, 414 (2005).

21. A. Umar, B. Karunagaran, E. -K. Suh and Y. B. Hahn, Nanotechnology 17, 4072 (2006).

22. Y. -S. Ho and K. -Y. Lee, Thin Solid Films 519, 1431 (2010).

23. J. H. Noh, I. -S. Cho, S. Lee, C. M. Cho, H. S. Han, J. -S. An, C. H. Kwak, J. Y. Kim, H. S. Jung, J. -K. Lee and K. S. Hong, phys. status solidi A-Appl. Mat. 206, 2133 (2009).

24. J. Cui, J. Phys. Chem. C 112, 10385 (2008).

25. B. Cao, W. Cai and H. Zeng, Appl. Phys. Lett. 88, 161101 (2006).

26. D. C. Look, D. C. Reynolds, C. W. Litton, R. L. Jones, D. B. Eason and G. Cantwell, Appl. Phys. Lett. 81, 1830 (2002).

27. S. Kim, M. S. Kim, H. Park, G. Nam, H. Yoon and J. -Y. Leem, Electron. Mater. Lett. 10, 3 (2014).

28. H. Ding, Z. Zhao, G. Zhang, Y. Wu, Z. Gao, J. Li, K. Zhang, N. Pan and X. Wang, J. Phys. Chem. C 116, 17294 (2012).

29. G. Nam, B. Kim, Y. Park, S. Park, J. Moon, D. Y. Kim, S. -O Kim and J. -Y. Leem, J. Mater. Chem. C 2, 9918, (2014).

30. N. Kumar, R. Kaur and R. M. Mehra, J. Lumin. 126, 784 (2007). 